\title{
ESTADO DA ARTE DAS WAVELETS: UMA ABORDAGEM DA APLICAÇÃO DE WAVELETS COMO FERRAMENTA DE PROCESSAMENTO GEOFÍSICO NA ATIVIDADE PETROLÍFERA
}

\author{
D. F. LIMA', M. M. N. SILVA², A. M. SOUSA JUNIOR ${ }^{1,2^{*}}$ e L. S. LUCENA² \\ ${ }^{1}$ Universidade Federal Rural do Semi-Árido - UFERSA \\ ${ }^{2}$ Universidade Federal do Rio Grande do Norte - UFRN \\ almir.mariano@ufersa.edu.br*
}

Artigo submetido em janeiro/2016 e aceito em março/2016

DOI: $10.15628 /$ holos.2016.3927

\section{RESUMO}

A investigação do subsolo, que tem como objetivos principais identificar condições geológicas e jazidas, necessita de métodos efetivos para que sejam obtidos resultados compatíveis com a realidade do campo. Nesta perspectiva, utilizar ferramentas que auxiliem no processo de análise dos estudos realizados in loco são imprescindíveis para que sejam auferidas interpretações mais precisas. Em virtude da heterogeneidade multiescala dos reservatórios de petróleo, a obtenção de propriedades equivalentes das formações rochosas mais finas resulta em uma problemática, pois elas são variáveis. Nesse sentido, as Wavelets são funções desenvolvidas através de diversas áreas: a matemática, a física quântica, a engenharia e a geologia, que permitem o tratamento de dados coletados na área de estudo e conferem um teor de maior concisão à pesquisa, visto que por meio delas acontece a decomposição multiescala e, consequentemente, uma adequação no processamento de propriedades de rochas e fluxo em um reservatório com heterogeneidades em várias escalas. Esse trabalho tem como finalidade analisar a utilização das Wavelets no processo de investigação do subsolo, mais especificamente na detecção de hidrocarbonetos. Para isso, foi feita uma pesquisa bibliográfica dos assuntos inerentes à temática e considerados os elementos relevantes para esta pesquisa. Verifica-se que as Wavelets contribuem para a efetividade das investigações geofísicas, tendo grande aplicabilidade na exploração petrolífera, uma vez que possibilita a descrição de condições geológicas de intervenção de maneira mais segura, e garantindo melhores resultados de aferições.

PALAVRAS-CHAVE: Investigação do subsolo, Wavelets, Sísmica.

\section{STATE OF THE ART OF WAVELETS: AN APPLICATION OF THE APPROACH OF WAVELETS AS A GEOPHYSICIST IN THE PETROLEUM PROCESSING TOOL ACTIVITY}

\section{ABSTRACT}

The investigation of the underground, which has as main objective to identify geological conditions and deposits, needs effective methods so that results obtained are consistent with the reality of the field. In this perspective, using tools that assist in the review process of the studies conducted on site are essential for them to be earned more accurate interpretations. Due to the heterogeneity of multiscale oil reservoirs, to obtain properties equivalent of thinner formations results in a problem because it is variables. In this sense, Wavelets are developed functions through several areas: mathematics, quantum physics, engineering and geology, which allow the processing of data collected in the study area and give a greater brevity content research, since by among them
\end{abstract}

happens multiscale decomposition and, consequently, an adjustment in the processing of rock properties and flow in a reservoir heterogeneities in several scales. This work aims to analyze the use of wavelets in the subsoil investigation process, specifically in hydrocarbon detection. For this, a literature search was made of the issues inherent to the theme and considered relevant for this research. It is found that Wavelets contribute to the effectiveness of geophysical investigations, with great applicability in oil exploration, as it enables the description of geological conditions more safely intervention, and ensuring better results of measurements. 


\section{INTRODUÇÃO}

A investigação do subsolo é imprescindível para identificação das características geológicas de uma determinada porção de terra em estudo, o que vai determinar as propriedades que são propícias para retenção de diversos materiais, dentre eles os hidrocarbonetos.

Há dois tipos principais de métodos de análise da subsuperfície: métodos diretos ou mecânicos; métodos indiretos ou geofísicos. Os métodos diretos baseiam-se na extração de amostras, enquanto os indiretos estudam o subsolo através de observações.

Quando se trata de investigação de petróleo, os métodos geofísicos apresentam-se como uma alternativa mais viável em virtude de serem mais econômicos do que os mecânicos. Nesse contexto, o método indireto sísmico, que permite a representação do perfil geológico por meio de sismogramas é muito utilizado neste tipo de pesquisa.

Como ferramenta auxiliadora do detalhamento com maior precisão dos estudos geofísicos, as Wavelets se apresentam eficazes, pois conforme Protázio (2002), elas podem ser definidas como funções matemáticas que separam um sinal em suas componentes de diferentes frequências e estudam cada uma das componentes com a resolução compatível para esta escala.

Além disso, de acordo com Protázio (2002) as Wavelets possibilitam o tratamento de dados coletados por meio de métodos de investigação do subsolo, conferindo maiores exatidões, já que os reservatórios de petróleo detêm uma heterogeneidade multiescala, o que dificulta a obtenção de propriedades equivalentes, e por meio das Wavelets ocorre a decomposição multiescala para que essas características sejam melhores definidas.

Quando se fala em métodos sísmicos, a transformada Wavelet permite efetuar a transferência de escala, ou seja, filtragem das informações geológicas, reduzindo-as às bandas de frequência da sísmica e, por consequência, obtendo-se sismogramas sintéticos. Isto é, são alcançados resultados com maior precisão para que as conclusões relacionadas com a situação real sejam coerentes.

Este trabalho tem como objetivos apresentar os métodos de investigação do subsolo, expor estudo da arte acerca das Wavelets e discutir as funções Wavelets na análise do método sísmico.

\section{MÉTODOS DE INVESTIGAÇÃO DO SUBSOLO}

De acordo com Chiossi (2013) a investigação do subsolo, que possibilita a identificação das condições geológicas da subsuperfície, pode ser realizada através de dois métodos principais: geofísicos ou indiretos; mecânicos ou diretos. Os métodos geofísicos analisam o subsolo por meio da observação do terreno ou mesmo no ar através de campos de força, produzidos de forma natural ou artificial. Já os métodos mecânicos investigam o subsolo por extração de amostras.

Os métodos indiretos podem ser classificados conforme Tabela 1. 
Tabela 1 - Classificação dos métodos geofísicos

\begin{tabular}{|c|c|c|c|}
\hline Métodos & Campos de Força & Propriedades físicas & $\begin{array}{c}\text { Campos principais de } \\
\text { aplicação }\end{array}$ \\
\hline Gravimétricos & $\begin{array}{c}\text { Campo gravitacional } \\
\text { terrestre }\end{array}$ & Densidade & Pesquisa de petróleo \\
\hline Magnéticos & $\begin{array}{l}\text { Campo magnético } \\
\text { terrestre }\end{array}$ & $\begin{array}{l}\text { Suscetibilidade } \\
\text { magnética }\end{array}$ & Mineração \\
\hline Elétricos & $\begin{array}{c}\text { a) campo eletrico } \\
\text { natural; } \\
\text { b) campo elétrico } \\
\text { artificial }\end{array}$ & $\begin{array}{l}\text { a) condutividade } \\
\text { elétrica; } \\
\text { b) condutividade ou } \\
\text { resistividade elétrica }\end{array}$ & $\begin{array}{c}\text { Água subterrânea e } \\
\text { engenharia civil }\end{array}$ \\
\hline Sísmicos & $\begin{array}{l}\text { Campo de vibração } \\
\text { elástica }\end{array}$ & $\begin{array}{l}\text { Velocidade de } \\
\text { propagação de ondas } \\
\text { elásticas }\end{array}$ & $\begin{array}{c}\text { Petróleo e engenharia } \\
\text { civil }\end{array}$ \\
\hline
\end{tabular}

Fonte: Chiossi (2013). Adaptado.

Quanto aos principais tipos de métodos diretos, podemos citar: manuais (poços, trincheiras, trado manual simples, sonda empire) e mecânicos (sondagens à percussão, sondagem à jato d'água, sondagem rotativa com extração de testemunho, sondagem rotativa sem extração de testemunho). Eles permitem, entre outros fins, definir o comportamento geológico do subsolo, extrair matérias primas e detectar medidas necessárias para que um trabalho seja executado com qualidade, por exemplo, rebaixamento do lençol freático e ventilação de minas.

Neste trabalho apresentaremos com mais detalhes os métodos geofísicos ou indiretos, especialmente aqueles que são mais utilizados para extração de petróleo (gravimétrico e sísmico), dando maior ênfase ao sísmico.

Conforme mostra a Tabela 1, o método gravimétrico consiste em investigar as estruturas geológicas através do campo gravitacional de determinada região ou local. Nesse método é imprescindível conhecer a densidade das rochas, que variam de acordo com sua composição, visto que quando houver distorção na distribuição lateral de densidades, a gravidade também apresentará irregularidades.

Já os métodos sísmicos, conforme Chiossi (2013), utilizam duas características importantes das formações rochosas: a velocidade de propagação de ondas elásticas varia de acordo com a tipologia da rocha e está vinculada às propriedades elásticas do material; os limites (contatos) que separam diferentes tipos de rocha refletem e refratam parte da energia das ondas elásticas.

No caso de utilização do método sísmico por reflexão, explosões são geradas propositalmente para fins de análises e estas resultam ondas de propagação no subsolo. A captação das ondas sísmicas é feita por meio de geofones e hidrofones, e os sismômetros são equipamentos utilizados para mensurar as reflexões e converter as velocidades de partículas ou variações de pressões em tensões e, assim, as análises necessárias podem ser realizadas.

Para interpretar os dados sísmicos são elaboradas representações dos sismogramas no intuito de serem detectadas as estruturas geológicas existentes.

Dessa forma, conforme Ecco (2011), a localização de jazidas é o grande objetivo quando se fala em exploração de hidrocarbonetos, sendo necessário associar as melhores formas de identificá-las à otimização do custo-benefício da extração de petróleo. Nesta perspectiva, os 
métodos sísmicos são os mais utilizados tendo em vista que sondam as camadas da superfície sem invadi-las, ou seja, analisam o ambiente de forma indireta e, consequentemente são mais econômicos se comparados aos métodos diretos de perfuração de poços.

\section{ESTADO DA ARTE ACERCA DAS WAVELETS}

De acordo com Kumar (1994), em 1910, o físico Alfred Haar desenvolveu um sistema completo de funções ortogonais com muitas propriedades e características que fazem das Wavelets uma das ferramentas matemáticas com um abrangente campo de aplicações. Posteriormente, ainda conforme Kumar (1994), as pesquisas acerca das Transformadas de Wavelet foram intensificadas na década de 80 através de artigos escritos pelo geofísico francês Jean Morlet e na segunda metade da década de 80 que foram estabelecidos os conceitos que permitiam compreender claramente essas funções, estabelecendo as suas propriedades e permitindo as construção e geração de outras wavelets.

Após algumas décadas e aprofundamentos matemáticos as transformadas de Wavelets ganharam aplicabilidade nas análises geofísicas. Assim, Lima (2003) traz uma abordagem teórica das Wavelets e as apresentam como ferramentas de grandes potencialidades e aplicabilidades nas mais diversas áreas. Nesse sentido, as Wavelets são definidas por Protázio (2002) como funções matemáticas que separam um sinal em suas componentes de diferentes frequências e estudam cada uma das componentes com a resolução compatível para esta escala.

Protázio (2002) afirma ainda que este método apresenta melhor aplicabilidade quando comparado à Transformada de Fourier na análise de situações físicas onde o sinal contém descontinuidades e pulsos.

Astaf'eva (1996) pontua que uma considerável parte da teoria das Wavelets foi desenvolvida de forma independente em vários campos científicos, dentre eles: a matemática, a física quântica, a engenharia e a geologia. As contribuições dadas auxiliam na ampliação de suas aplicações, tornando-se possível realizar processamentos e compressão de imagens, radares, entre outras.

Desse modo, Daubechies (1998) diz que a teoria da Transformada Wavelet é apresentada e mostra-se uma ferramenta adequada e bem adaptada para a investigação e análise de processos não estacionários envolvendo um grande número de escalas. Diferentemente da Transformada de Fourier, a Transformada Wavelet é bem localizada tanto no tempo quanto na frequência.

Conforme Prokoph e Barthelmes (1996), a análise obtida através da Transformada Wavelet foi utilizada na detecção e localização de descontinuidades, eventos e sequências cíclicasperiódicas e caóticas em uma sucessão sedimentar marinha. A Transformada Wavelet com base em Kumar (1994) também foi adotada na localização exata das transições e mudanças das sucessões sedimentares, permitindo dessa forma uma localização simplificada a partir do conjunto de dados.

Já no trabalho de Mallat (1996), a análise do espectro obtido através da Transformada Wavelet foi aplicada a um conjunto de dados de ventos oceânicas, coletados durante o programa Surface Wave Dynamics Experiment - SWADE, de 1990 e resultados novos foram obtidos. Tais 
resultados não eram evidentes quando as análises eram desenvolvidas a partir da Transformada de Fourier.

Em 1996, Chu (1996) trouxe uma nova metodologia para a contagem de pixels que se mostrava mais eficiente do que as numerosas técnicas já existentes. Neste caso, também eram adotadas as Wavelets para o processamento de imagens.

A aquisição de métodos precisos e eficientes para a obtenção das propriedades equivalentes das rochas dos reservatórios em escalas mais finas se apresentam como uma grande problemática. Devido a natureza das heterogeneidades multiescalas resultantes dos reservatórios de petróleo, as propriedades das rochas e do fluido equivalentes variam. Sendo assim, a análise realizada através da Transformada Wavelet é um método de decomposição multiescala e se adequada no processamento de propriedades de rochas e fluxo em um reservatório com heterogeneidades em várias escalas.

A Figura 1 mostra uma Wavelet em três escalas diferentes.

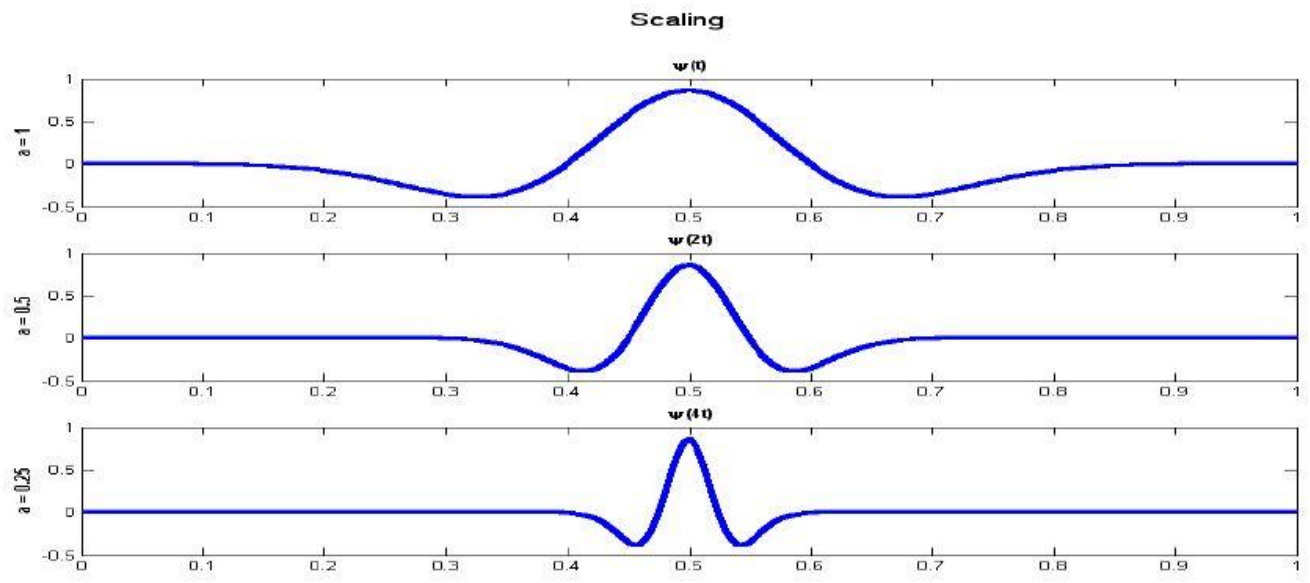

Figura 1 - Wavelet em três escalas.

Fonte: Protázio (2002).

Nesse sentido, os trabalhos de Kumar (1994) mostra que o estudo de propriedades petrofísicas gerais é necessário, pois importantes informações sobre o reservatório são obtidas em escalas mais finas que os blocos de discretização utilizados para simular o reservatório.

Em 1998, Jansen (1998) aponta que modelos geológicos gerados em uma escala fina determinam modelos de reservatório contendo centenas de milhares de blocos de dados a serem processados e mesmo para computadores de alto desempenho, este tipo de abordagem se apresentava muito demorada, o que inviabilizava a sua utilização na simulação de reservatórios. Partindo dessa discussão, a Transformada Wavelet tem a capacidade de preservar estruturas locais em conjuntos de dados espaciais e para reduzir os esforços computacionais, estas foram empregadas nas atividades de processamento das imagens obtidas a partir das sísmicas, em uma escala maior, com duas ou três dimensões.

Em 1997, Torrence \& Compo (1997), discutem a partir de uma comparação do método com a Transformada de Fourrier de curta duração - WFT, a escolha da função Wavelet apropriada, os 
efeitos de borda devido ao comprimento finito das séries temporais e a relação entre as escalas Wavelet e a frequência de Fourier. Ainda nesse período, Jansen (1997) aponta que os dados de produção são uma das maiores fontes de informação disponíveis sobre o comportamento do reservatório e do poço. Ressalta-se também que a correlação cruzada entre pares de poços pode fornecer informações acerca da direção do fluxo em um reservatório. Entretanto, os cálculos referentes a essa direção são muito complexos. Isto se deve principalmente à natureza não linear e não estacionária das relações entre poços. A relação entre poços é uma função das condições impostas pelas propriedades do reservatório.

Partindo dessa discussão, a Transformada Wavelet é mostrada por Hagelberg e Gamage (1994) como uma nova ferramenta, que diferente da Transformada de Fourier, permite um tratamento de dados não estacionários. Tal fator aponta novas possibilidades quanto ao que se refere às correlações mais complexas entre poços e a utilização destes dados para uma determinação mais consistente das causas do comportamento do poço e sua influência nos poços ao seu redor. Em contra partida, Johansson (2005) afirma que as análises obtidas através da Transformada Wavelet são sensíveis para detecção automática e distinção de descontinuidades, tais como falhas, descontinuidades, ciclicidades e mudanças graduais na taxa de sedimentação.

Em 2000, Oliver, Bosch \& Slocum (2009) aplicaram a decomposição através da Transformada Wavelet para descrever e localizar um amplo espectro de frequências simultaneamente e, então, filtrá-las através da decomposição multi-resolução obtida através da Transformada Wavelet. O método foi aplicado e comparado com a filtragem obtida pelo uso da Krigagem Fatorial, com resultados satisfatórios.

Silva (2001) mostra que nos métodos usuais utilizados na obtenção de sismogramas sintéticos, o sinal de perfil sofre uma conversão de escala do domínio do espaço para o domínio do tempo. Comumente, o controle da filtragem utiliza o sinal sísmico, com espectro eficiente e preciso, mas que, apresenta uma cobertura espacial mais adequada ao reconhecimento das informações geológicas.

\section{FUNÇÕES WAVELETS NA ANÁLISE DO MÉTODO SÍSMICO}

O método sísmico baseado na propagação de ondas elásticas, reflexão e refração, permite a elaboração do sismograma, ou seja, a representação das configurações do subsolo através de imagens, que podem ter resoluções diferentes de acordo com o procedimento de análise empregado.

Para Ecco (2011), a teoria das Wavelets reúne diversas técnicas elaboradas de forma independente para várias aplicações de processamento de sinais. Elas são funções que permitem representar uma função em resoluções diferentes além de comprimirem dados sísmicos nas pesquisas de petróleo.

Para entender melhor as Wavelets, devemos compreender o processo evolutivo de avaliação dos sinais estacionários e não estacionários emitidos por meio dos métodos geofísicos.

A transformada de Fourrier foi desenvolvida para analisar sinais estacionários, já que considera todo o espaço ao representar a transformada integral de função senoidal. Em virtude da 
necessidade do estudo dos sinais não-estacionários também, surge a transformada de Fourrier em janelas, que estuda os sinais em intervalos iguais. Porém devido essas janelas (larguras) serem iguais, é utilizada uma mesma janela independentemente da frequência. Assim, as transformadas Wavelets disponibilizam janelas de tamanhos variáveis, o que permite uma análise mais detalhada e precisa.

Por meio da transformada de Wavelets é possível efetuar a transferência de escala, ou seja, filtragem das informações geológicas, reduzindo-as às bandas de frequência da sísmica e, por consequência, obtendo-se sismogramas sintéticos.

Além disso, conforme Protázio (2002) a análise Wavelet pode ser aplicada na supressão de ruídos em uma imagem de atributos petrofísicos, ou seja, imagem sísmica. Para isso, é necessário realizar a decomposição multi-resolução no tratamento de supressão de ruído.

Dessa forma, a utilização de Wavelets é muito importante para que possam ser auferidas conclusões coerentes com as características reais do espaço que se deseja investigar.

\section{CONSIDERAÇÕES FINAIS}

As análises geofísicas ajudam a descrever o comportamento geológico do subsolo e a partir daí se tornam indispensáveis para a realização de inúmeras atividades com fins exploratórios. Dentre estas, destaca-se a exploração do petróleo, onde comumente são realizadas investigações a partir de sísmicas de refração ou reflexão para que seja possível detectar a existência de hidrocarbonetos e assim, definir a viabilidade da exploração.

Nesse sentido, o processamento das sísmicas contribui para a eficiência das análises e bom desempenho das atividades seguintes e alguns métodos podem ser implementados para facilitar esta etapa. Dentre eles, podemos citar a Transformada de Fourrier, que consiste em um método de modelagem matemática, entretanto, tem uma eficiência reduzida quando se trata de uma área com um comportamento descontínuo, visto que a partir daí formam-se pulsos e esta ferramenta não consegue processá-los de forma precisa.

Assim, as Wavelets ganham uma grande aplicabilidade nesse contexto, uma vez que a partir destas torna-se possível descrever as condições geológicas das áreas de intervenção de forma mais segura e propiciando melhores resultados na exploração petrolífera, já que elas permitem a supressão de ruídos e o melhoramento da qualidade gráfica de uma imagem sísmica com a realização de decomposição multiresolução.

\section{REFERÊNCIAS}

1. ASTAF'EVA, N. M. Wavelet analysis: basic theory and some applications. Disponível em: < http://iopscience.iop.org/article/10.1070/PU1996v039n11ABEH000177/pdf>. Acesso em: 25 dez. 2015.

2. BOSCH, B. E.; GONZÁLEZ, P. A.; VIVAS, G. J. Directional wavelets and a wavelet variogram for two-dimensional data. 2009. Disponível em: <http://repositorio.ufba.br/ri/handle/ri /13124>. Acesso em: 25 dez. 2015. 
3. CHIOSSI, N. J. Geologia Aplicada à Engenharia. 3a ed. São Paulo: Oficina de Textos, 2013.

4. CHU. Lifu. Application of Wavelet Analysis to Upscaling of Rock Properties. Disponível em: < https://www.onepetro.org/journal-paper/SPE-36517-PA>. Acesso em: 25 dez. 2015.

5. DAUBECHIES, Ingrid. Orthonormal Bases of Compactly Supported Wavelets. 1998. Disponível em: < http://cbio.ensmp.fr/ jvert/svn/bibli/local/Daubechies19880rthonor mal.pdf>. Acesso em: 25 dez. 2015.

6. ECCO, D. Remoção de ruídos sísmicos utilizando transformada Wavelet 1D e 2D com software em desenvolvimento. Dissertação de Mestrado. Natal/RN, abr. 2011.

7. HALGelberG, Carl R.; GAMAGE, Nimal K. K. Aplications Of Estrutcuture Preseving Wavelets Decompositions to Intermitent Turbulence: a case study. Disponível em: < https://books. 20Multi-scale\%20information\%20in\%20thecharacterizationof\%20hydraulic \%20conductivitydistributions\&f=false>. Acesso em: 25 dez. 2015.

8. JANSEN, F. E. Upscaling of Reservoir Properties Using Wavelets. Disponível em: < https://www.onepetro.org/conference-paper/SPE-39495-MS>. Acesso em: 25 dez. 2015.

9. JOAHNSSON. Elin. Wavelet Theory and some of its Applications. Disponível em: < http://epubl.Itu.se/1402-1757/2005/10/LTU-LIC-0510-SE.pdf>. Acesso em: 25 dez. 2015.

10. KUMAR. Praveen. Wavelets in Geopphysics. Disponível em:< https://books. scale\%20information\%20in\%20thecharacterizationof\%20hydraulic\%20conductivitydistrib utions\&f=false $>$. Acesso em: 25 dez. 2015.

11. LIMA, Paulo Cupertino de. Wavelets: uma introdução. Disponível em:< http://www.mat.ufmg.br/ lima/artigos/rmu33.pdf >. Acesso em: 25 dez. 2015.

12. MALLAT, Stephany. A Theory for Multiresolution Signal Decomposition: The Wavelet Representation. Disponível em: < http://www.cmap.polytechnique.fr/ mallat/papiers/Ma llatTheory89.pdf>. Acesso em: 25 dez. 2015.

13. PROKOPH, Andreas; AGTERBERG, Frederik P. Wavelet analysis of well-logging data from oil source rock, Egret Member, offshore eastern Canada. Disponível em: < http://archives.datapages.com/data/bulletns/2000/10oct/1617/1617.htm>. Acesso em: 25 dez. 2015.

14. PROTÁZIO, J. M. B. Análise Wavelets aplicada a sinais geofísicos. Dissertação de Mestrado. Campinas/SP, abr. 2002.

15. SILVA, Adalberto da. Análise de perfis geofísicos de poço no domínio de Fourier e sua integração com a aquisição sísmica no modelamento de reservatórios. 2001. Disponível em: <http://www.bv.fapesp.br/pt/bolsas/43295/analise-de-perfis-geofisicos-de-poco-nodominio-de-fourier-e-sua-integracao-com-a-aquisicao-sismica/>. Acesso em: 25 dez. 2015. 